\title{
Functional genomics to explore cancer cell vulnerabilities
}

\author{
*Kristopher T. Kahle, M.D., Ph.D., ${ }^{1-4}$ David Kozono, M.D., Ph.D.,, 6 \\ Kimberly Ng, B.S., ${ }^{6}$ Grace Hsieh, B.A., ${ }^{6}$ Pascal O. Zinn, M.D., ${ }^{6}$ \\ Masayuki Nitta, M.D., Ph.D., ${ }^{6}$ and Clark C. Chen, M.D., Ph.D. 6 ,7 \\ ${ }^{1}$ Department of Neurosurgery, Massachusetts General Hospital; ${ }^{2}$ Howard Hughes Medical Institute; \\ ${ }^{3}$ Department of Genetics, Harvard Medical School; ${ }^{4}$ Department of Medicine and ${ }^{5}$ Harvard Radiation \\ Oncology Program, Brigham and Women's Hospital; ${ }^{6}$ Department of Radiation Oncology, \\ Dana-Farber Cancer Institute; and ${ }^{7}$ Division of Neurosurgery, Beth Israel Deaconess Medical Center, \\ Boston, Massachusetts
}

\begin{abstract}
Our understanding of glioblastoma multiforme (GBM), the most common form of primary brain cancer, has been significantly advanced by recent efforts to characterize the cancer genome using unbiased high-throughput sequencing analyses. While these studies have documented hundreds of mutations, gene copy alterations, and chromosomal abnormalities, only a subset of these alterations are likely to impact tumor initiation or maintenance. Furthermore, genes that are not altered at the genomic level may play essential roles in tumor initiation and maintenance. Identification of these genes is critical for therapeutic development and investigative methodologies that afford insight into biological function. This requirement has largely been fulfilled with the emergence of RNA interference (RNAi) and high-throughput screening technology. In this article, the authors discuss the application of genome-wide, highthroughput RNAi-based genetic screening as a powerful tool for the rapid and cost-effective identification of genes essential for cancer proliferation and survival. They describe how these technologies have been used to identify genes that are themselves selectively lethal to cancer cells, or synthetically lethal with other oncogenic mutations. The article is intended to provide a platform for how RNAi libraries might contribute to uncovering glioma cell vulnerabilities and provide information that is highly complementary to the structural characterization of the glioblastoma genome. The authors emphasize that unbiased, systems-level structural and functional genetic approaches are complementary efforts that should facilitate the identification of genes involved in the pathogenesis of GBM and permit the identification of novel drug targets. (DOI: 10.3171/2009.10.FOCUS09212)
\end{abstract}

\section{Key Words - functional genomics - RNA interference • short interfering RNA • glioblastoma multiforme • cancer}

$\mathrm{G}$ LIOBLASTOMA multiforme is the most common and lethal of all primary brain tumors. It is diagnosed in approximately 10,000 new patients each year in the US. Despite its comparatively low incidence of about 2-3 new cases per 100,000 people per year (for comparison, the incidence of colon cancer in the US is approximately 1 new case per 1800 people per year), the total number of deaths per year attributable to GBM rivals that of other major cancers. Diffuse invasion into the surrounding brain is a cardinal feature of GBM, essentially preventing surgical cure and complicating the delivery of therapeutic agents. ${ }^{14,15}$ Uniformly - if untreated-patients with GBM die within 3 months of their diagnosis. Current therapies, while prolonging overall survival, remain largely palliative, with most patients succumbing to

\footnotetext{
Abbreviations used in this paper: $\mathrm{GBM}=$ glioblastoma multiforme; MOI = multiplicity of infection; RISC = RNA-induced silencing complex; RNAi = RNA interference; shRNA = RNA short hairpin; siRNA = short interfering RNA.

* Drs. Kahle and Kozono contributed equally to this manuscript.
}

their disease within 1 year of diagnosis. Novel targeted therapeutic approaches are desperately needed.

As with many other types of cancer, a major bottleneck in devising effective targeted therapies for GBM lies in the identification of relevant drug targets. For a given tumor, the choice of targets will depend on the particular genetic network that supports its cancerous phenotype. The identification of genes mutated in human cancer has resulted in novel, pathogenesis-oriented treatment strategies. Gain-of-function mutations in oncogenes represent promising targets for future drug development because many tumors, via a dependency termed "oncogene addiction," rely on these genetic alterations for survival. ${ }^{32}$ Substantial efforts have been devoted to the development of cancer therapeutics targeting oncogenes. Notable successes that have resulted from this strategy include the tyrosine kinase inhibitor Gleevec (which blocks the $B C R$ $A b l$ gene fusion product) in the treatment of leukemias, and erlotinib and gefitinib (EGFR inhibitors) in the treatment of non-small cell lung cancers harboring activating EGFR mutations. ${ }^{8}$ The clinical utility of these drugs has reinforced the notion that a more thorough understanding 
of the molecular networks governing cancer pathogenesis can be translated into substantial clinical benefits.

However, human cancers harbor hundreds of genetic alterations, and only a subset of these alterations likely drive tumor initiation and maintenance, making it difficult to identify which mutations are the critical drivers of cell transformation, and how multiple mutations within single cells interact to promote tumorigenesis. Furthermore, genes not altered at the genomic level play essential roles in tumor development, in so-called "nononcogene addiction." ${ }^{29}$ For instance, cancer cells exhibit increased dependence on the heat shock response, although genes required for this response are not oncogenic. ${ }^{35}$

Given the limitations inherent within the genomic approach, a systematic functional assessment of the contribution of specific genes to cancer phenotypes is an alternative but complementary approach to structural characterizations of the cancer genome. The recent development of genome-wide human RNAi libraries has enabled systematic genetic studies in normal and human breast and colon tumor cells using arrayed and pooled screens. ${ }^{3-5,12,19,24,27,33}$ It seems likely that systematic RNAi screens aimed at generating either cancer lethal signatures using a large panel of cancer and normal human cell lines, or synthetic lethal signatures against specific oncogenes or tumor suppressors or cells, should reveal the key glioma cell vulnerabilities that could serve as the basis for the rational design of novel therapeutics.

\section{Mutational Analysis of the GBM Genome}

A traditional approach used to discover novel drug targets is to identify genetic lesions in cancer cells and exploit this knowledge for therapeutics. Such an approach is currently used by the Cancer Genome Atlas and other similar efforts that aim to characterize-in a large number of cancer types-genomic alterations, including copynumber variation, transcriptional profiles, epigenetic modifications, and DNA sequence alterations. This approach has the potential to identify common alterations in oncogenes and tumor suppressors for further functional analysis and to uncover oncogene addiction pathways that can be targeted. ${ }^{7,21}$ For example, Yan et al., ${ }^{36}$ by sequencing 20,661 protein-coding genes, determining the presence of amplifications and deletions using high-density oligonucleotide arrays, and performing gene expression analyses using next-generation sequencing technologies in 22 human GBM samples, found alterations in a variety of previously known and unknown GBM-associated genes. Most notably, novel mutations in the active site of isocitrate dehydrogenase 1 (IDH1), were demonstrated in $12 \%$ of patients with GBM; most of these mutations occurred in a large fraction of young patients and in most patients with secondary GBMs and were associated with an increase in overall survival.

In the Cancer Genome Atlas study, besides recapitulating the mutational events in GBM that have been known for decades on a large scale, the authors found that some genetic lesions-such as TP53 mutation, NF1 deletion or mutation, and ERBB2 amplification-were more common than previously reported.7 Together, these systems- level studies identified the major genetic events in human GBMs, ${ }^{7,21}$ including: 1) dysregulation of growth factor signaling via amplification and mutational activation of receptor tyrosine kinase (RTK) genes; 2) activation of the phosphatidylinositol-3-OH kinase (PI(3)K) pathway; and 3 ) inactivation of the p53 and retinoblastoma ( $\mathrm{Rb})$ tumor suppressor pathways. Recent genome-wide profiling studies have also demonstrated the remarkable genomic heterogeneity among GBMs, along with the existence of different molecular subclasses of GBMs that may allow for treatment stratification. ${ }^{13,22}$ However, with rare exceptions, such as $I D H I$, few of the discoveries from these structural studies are anticipated to generate immediately actionable drug targets; for common but currently undruggable oncogenes such as $R A S$, and tumor suppressors, such as PTEN and TP53, the Cancer Genome Atlas will likely provide little translational insight.

\section{Limitations of Structural Analyses of Cancer Genomes for Drug Discovery}

Although informative, structural genetic approaches have limitations with respect to target discovery. First, current sequencing capacity is largely limited to coding regions, though whole-genome sequencing will become feasible with the development of cheaper and more highly parallel sequencing methods. Second, targeting oncogene addiction alone is unlikely to be a solution because: 1) many known oncogenes are challenging therapeutic targets (for example, efforts to develop drugs that inhibit oncogenic $R A S$ proteins have been largely unsuccessful, despite the fact that $R A S$ is mutated in nearly $30 \%$ of human tumors and is essential for their viability); 2) although recent sequencing/mapping efforts have revealed hundreds of potential oncogenic mutations and gene copy alternations, without functional characterization it is unknown which of these mutations make good drug targets; and 3) because tumors rapidly develop resistance to targeted therapy with single agents, multiple agents with different mechanisms of action are necessary to suppress drug resistance.

In addition to oncogene addiction, the stress phenotype of cancer cells makes them more dependent on the function of certain essential genes that are not themselves oncogenes or otherwise mutated; however, many if not all of these proteins can be rate-limiting to their pathways. Elledge and colleagues coined this phenomenon of cancer cells the "non-oncogene addiction." ${ }^{29}$ The cancer cells' increased dependence on the heat shock response and their metabolic adaptation for aerobic glycolysis are 2 examples of non-oncogene addiction that have received therapeutic attention. ${ }^{6,35}$ Because genes involved in nononcogene addiction do not themselves harbor genetic alterations, they will be missed by DNA sequencing efforts alone. Therefore, the identification of non-oncogene addiction genes requires functional approaches. ${ }^{29}$

\section{Functional Genomics: An Alternative Method for Exploring Cancer Cell Vulnerabilities}

A second approach takes that of functional systems 


\section{Functional genomics}

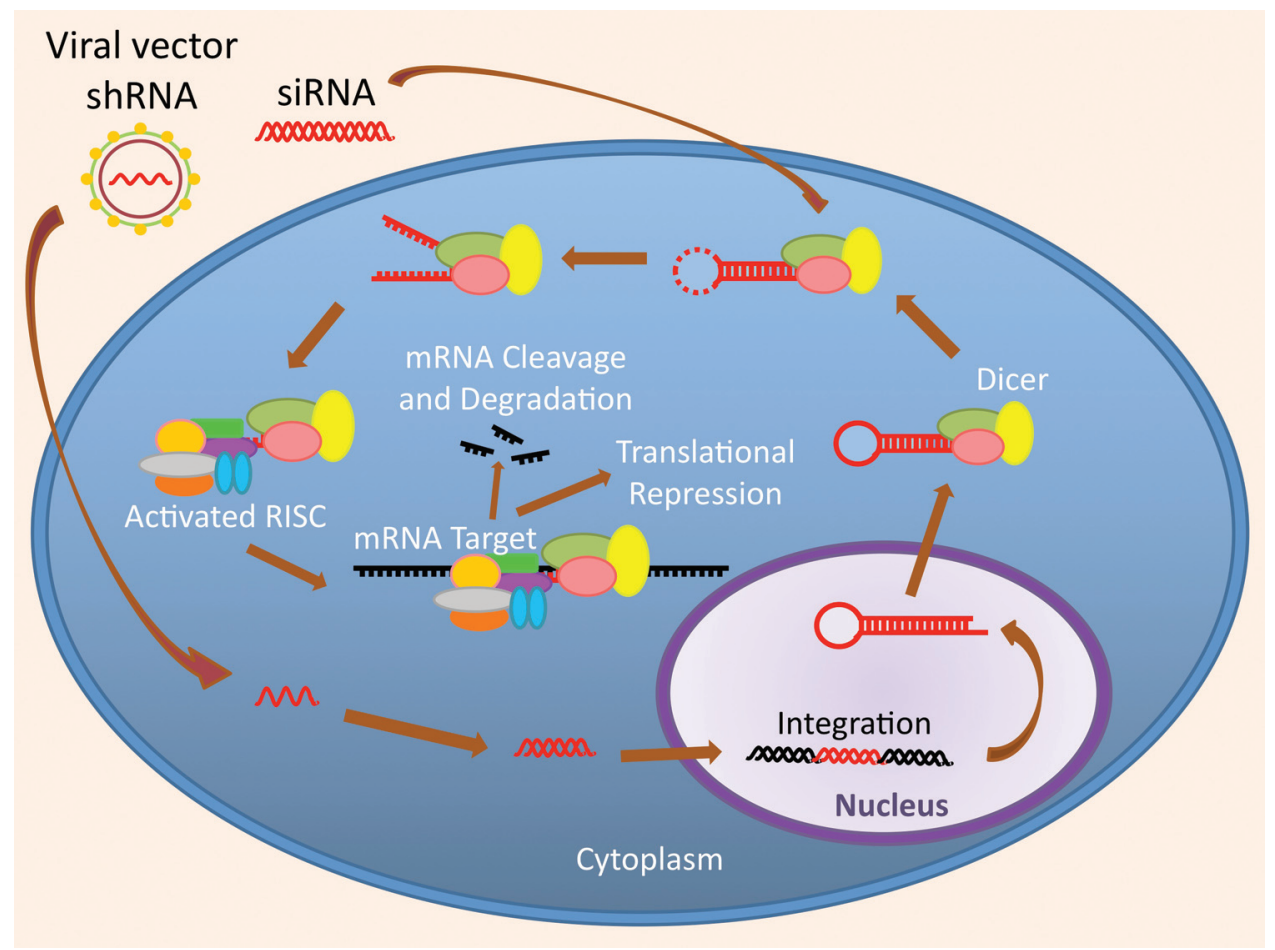

FIG. 1. The RNA interference pathway. Gene knockdown can be readily achieved in mammalian cells by transient transfection with chemically synthesized siRNAs or stable transfection with plasmids or viruses expressing shRNAs. In the latter case, the shRNA sequences integrate into the host genome and are expressed via Pol II- or Pol III-mediated transcription. The shRNAs are then processed by Dicer into double-stranded siRNA. Via either mechanism, the resulting siRNA becomes incorporated into a RISC, which directs target mRNA degradation or translational suppression in a sequence-specific manner, thus resulting in decreased protein expression.

biology to identify cancer cell vulnerabilities. ${ }^{19,24,27}$ Advances in RNAi technology in human cancer cell lines have made it possible to systematically interrogate genes for potential roles in tumorigenesis. By silencing or decreasing the expression of specific gene products in cancer-specific assays, these loss-of-function screens can provide insight into their biological function.

A functional genetic approach using genome-wide RNAi offers several advantages in target discovery over structural genomics: 1) RNAi-mediated protein knockdown results in either partial or complete loss of function, oftentimes mimicking the effect of a potential pharmacological inhibitor; 2) RNAi can be applied in an unbiased manner to any annotated gene in the human genome based on sequence information alone without a priori knowledge of the gene's mutational status or biological function, thereby helping uncover novel cancer genes; 3) RNAi can be readily applied in mammalian model systems (including the many different mouse models of cancer), thus providing an opportunity for rapid target validation in vivo without first developing a drug that inhibits the gene product of interest; 4) RNAi screening has the ability to identify genes not mutated in cancers and exhibit features of non-oncogene addiction.

It is possible to induce RNAi-mediated gene knockdown transiently by the introduction of chemically synthesized small interfering RNAs (siRNAs), or stably by plasmids or viruses expressing RNA short hairpins
(shRNAs), which are subsequently processed into siRNAs by the protein Dicer. Via either mechanism, the resulting siRNA directs degradation or translational suppression in a sequence-specific manner of the mRNA of the gene of interest by becoming incorporated into the RNA-induced silencing complex (RISC), resulting in decreased protein expression (Fig. 1). While chemically synthesized oligonucleotide siRNAs are effective in inducing gene suppression, such reagents are expensive and can only be used for transient loss-of-function experiments. This is limiting for many assays specific for cancer-related phenotypes, such as anchorage-independent colony formation or tumor xenografts, where long-term gene suppression is required. Vector-based RNAi systems have circumvented this problem by providing stable expression of the RNAi in mammalian cells through incorporation of the construct into the DNA of the host cell's genome. These vector-based systems also have the added benefit of being a renewable resource because of their propagation in Escherichia coli. Moreover, vector-based systems can be used to create retroviruses that harbor the RNAi construct, thereby expanding the type of cells into which such constructs can be introduced. ${ }^{20,23,26}$ Both siRNA and shRNA libraries have been used successfully in transfection-based arrayed screens investigating phenotypes that develop shortly after gene suppression, such as apoptosis, cell signaling events, or cell cycle distribution. ${ }^{1,11,34}$

The use of RNAi provides a straightforward, func- 


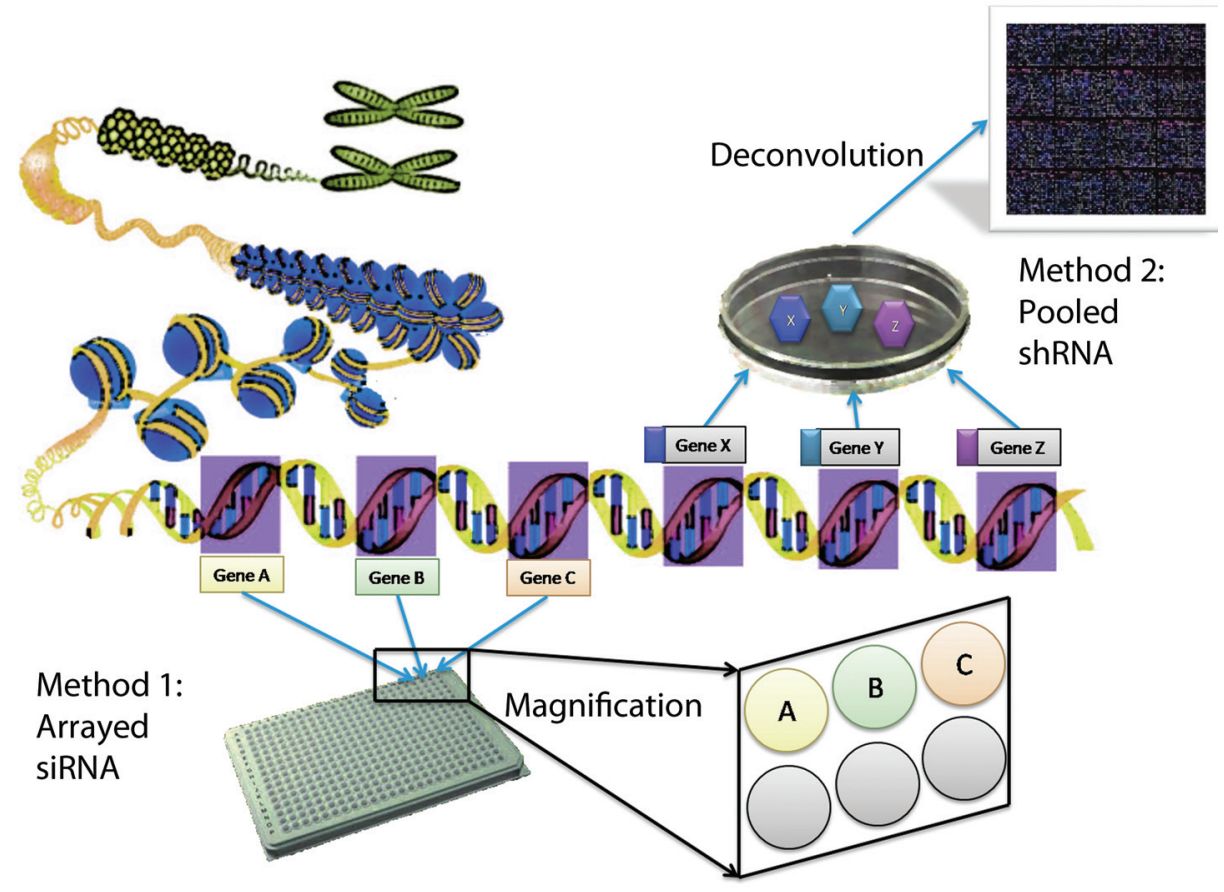

FIG. 2. Whole genome RNAi screening. The effect of gene knockdown of each of the approximately 25,000 genes of the human genome can be achieved using either of 2 general methods. In Method 1, siRNAs are arrayed into 96- or 384-well microtiter plates such that each well contains 1 siRNA directed against a single gene. After a few days, each well can be scored for readouts, such as cell viability, altered morphology, fluorescently tagged proteins, and so forth. In Method 2, thousands of different shRNA constructs that are each tagged with a unique barcode are used to transfect or infect pools of cells. Because these sequences integrate stably into the host genome, the effects of gene knockdown can be deconvoluted by barcode microarrays after several cell doublings.

tional approach to identify new cancer drug targets by identifying genes whose knockdown is lethal to cancer cells but not normal cells. To gain statistical power, one must screen multiple cancer and normal cell lines. Because of the sheer scale of screening that is required, the standard siRNA transfection or shRNA transduction screen in a well-by-well format using multiwell plates is prohibitively expensive and time consuming. Currently, several laboratories are equipped to execute such highthroughput approaches to manipulate gene expression in mammalian cell lines. However, novel technologies are developing that will make it cheaper and easier to execute such large-scale screens. Barcoded shRNA libraries, together with pool-based screening platforms, circumvent these barriers to large-scale screening (Fig. 2). Elledge and colleagues have pioneered the construction of shRNA libraries that target the human genome and have optimized delivery vectors that allow potent shRNA expression and efficient gene knockdown; these efforts have resulted in a barcoded, microRNA-based
shRNA library targeting the entire human genome that can be expressed efficiently from either retroviral or lentiviral vectors in a variety of cell types for stable gene knockdown. ${ }^{20,26,27}$ The current generation shRNA library consists of 87,283 shRNAs targeting 32,216 human genes, with an average of 3 distinct shRNA sequences per gene. ${ }^{18}$

There are 3 unique advantages of the Elledge-Hannon shRNA library: 1) The shRNA is embedded in the backbone of a naturally occurring microRNA, mir30, enabling efficient expression and processing of the shRNA for incorporation into the RISC (Fig. 3); 2) the shRNA of interest is cloned into a modified retroviral vector permitting sufficient expression so that single proviral integration is sufficient for gene knockdown-an essential characteristic for a pool-based, ultra high-throughput screening platform; and 3) each shRNA is tagged with a unique 60-nucleotide barcode sequence that can be used to track its abundance in a pool by microarray hybridization (Fig. 3). ${ }^{20,26,27}$ Elledge and colleagues have also developed an ultra high-throughput platform (see below) to

$$
\text { 5'-LTR } \psi \text { miR30-5, shRNA miR30-3' 60-nt barcode PGK Puro 3'-LTR }
$$

FIG. 3. Endogenous microRNA-based shRNA retroviral vector. This construct yields the advantage of sufficient gene knockdown following single-copy integration, which is essential for successful pooled screens. LTR = long terminal repeat; $\psi=$ psi retroviral packaging element; miR30 = human endogenous microRNA 30; PGK = phosphoglycerate kinase promoter; Puro = puromycin resistance gene. 
screen complex pools of shRNAs using barcodes coupled with microarray deconvolution. Barcodes are critical for pool-based dropout screens that are designed to identify cell-lethal or drug-sensitive shRNAs ${ }^{19}$ and provide independent means to track the abundance of shRNAs in a complex pool. Hairpins depleted over time can be identified through the competitive hybridization of barcodes derived from the shRNA population before and after selection to microarrays. ${ }^{21,27,28}$ This technology can handle more than 10,000 shRNAs per pool and involves low costs. ${ }^{27,34}$

\section{Cancer Lethal Signatures}

The central goal for developing pool-based shRNA screening platforms is to attain the means to rapidly perform dropout screens for the systematic identification of genes required for cancer cell proliferation and survival that could represent new drug targets. A proof-of-principle screen using the Elledge-Hannon shRNA library in human colon and breast cancer cells has recently been published. ${ }^{24}$

In their study, Schlabach et al. ${ }^{24}$ used a highly complex pool of 8203 distinct shRNAs targeting 2924 genes consisting of annotated kinases, phosphatases, ubiquitination pathway and cancer-related genes-all central regulators of signaling pathways that should provide a rich source of phenotypic perturbation-to interrogate multiple human colon cancer and breast cancer cell lines, and normal human epithelial cell lines. Two types of cancers with distinct origins were compared with identified common and cancer-specific growth regulatory pathways. Cells were infected with an average representation of 1000 per shRNA and an MOI of 1-2. Initial reference samples were collected 2-3 days postinfection; the remaining cells were puromycin-selected, propagated for several weeks, and collected again as the end samples. Polymerase chain reaction was used to recover the halfhairpin $(\mathrm{HH})$ barcodes from genomic DNA, labeled with $\mathrm{Cy} 5$ and $\mathrm{Cy} 3$ dyes, respectively, and hybridized to an $\mathrm{HH}$ barcode microarray. For each probe, the Cy3/Cy5 signal ratio reports the change in relative abundance of a particular shRNA between the beginning and the end of the experiment. An shRNA that causes growth inhibition or cell death will drop out from the pool over time and show a $\log _{2}$ Cy5/Cy3 ratio of $<0$. Conversely, a shRNA that enhances cell proliferation will enrich in the pool over time and show a $\log _{2} \mathrm{Cy} 5 / \mathrm{Cy} 3$ ratio of $>0$. Lastly, an shRNA that has little effect on cell growth will show a $\log _{2} \mathrm{Cy} 5 /$ $\mathrm{Cy} 3$ ratio of $\sim 0$. Whereas the majority of shRNAs show little changes in their abundance over time ( $\log _{2}$ ratio between -1 and 1), a small fraction of shRNAs dropped out over time $\left(\log _{2}\right.$ ratio $\left.<-1\right)$, reflecting differences between cancer and normal cells. Interestingly, the 2 colon cancer cell lines were more similar to each other than to the breast cancer line, likely reflecting tissue-specific differences and mechanisms of tumorigenesis.

As expected, the screen recovered multiple components of core cellular pathways essential for survival; for example, shRNAs against multiple subunits of the anaphase promoting complex (APC/C) and the eukaryotic translation initiation factor 3 (eIF3) complex were lethal in all cell types. In addition to the common set of shRNAs that impair viability in all cell lines, a number of genes selectively required for proliferation of individual cell lines were identified, including $P P P 1 R 12 A-$ a regulatory subunit of protein phosphatase 1; PPPIR12A was selectively required for breast cancer cell viability, but not colon cancer cell viability. Conversely, colon cancer cell lines were more sensitive to knockdown of PRPS2, a gene involved in nucleoside metabolism. Importantly, comparison of the lethal signatures between breast cancer cell lines and nontransformed human mammary epithelial cell lines identified a number of genes-including the cell cycle regulator kinase gene $B U B 1$-whose knockdown caused greater toxicity to breast cancer cells than normal mammary epithelial cells, suggesting $B U B I$ could be further explored as a potential drug target for the treatment of breast cancer.

This study proved the feasibility of ultra highthroughput dropout screens with highly complex pools of shRNAs with barcode microarray deconvolution to identify lethal shRNAs for multiple cancerous and normal epithelial cell lines. These studies resulted in the discovery of genes that are essential for general cell viability and are selectively required for cancer cell viability. Others have described the development and application of genome-scale high-throughput methods using lentiviral RNAi libraries to systematically assess cancer gene function and to integrate structural and functional approaches in the study of cancer. ${ }^{17}$

\section{Synthetic Lethal Signatures}

"Synthetic lethality" occurs when alteration of a gene results in cell death only in the presence of another genetic mutation. Cancer cells, due to oncogenic mutations, develop secondary dependencies on genes that are themselves not oncogenes. This fact is exploited in synthetic lethal genetic screens, where perturbation of genes within the same pathway, a parallel pathway, a distant pathway, or in genes with other essential cellular functions, result in oncogene-specific synthetic lethal interactions, thereby defining novel therapeutic targets. ${ }^{9}{ }^{910}$ Synthetic lethal interactions were first described in model organisms like yeast and worms, ${ }^{2,16}$ but recent studies have demonstrated the concept of synthetic lethality is relevant for mammalian cells. $^{28,30}$ The use of RNAi is also relevant for identifying genetic interdependencies in human cancer; functional genetic screens have identified genes whose knockdown sensitizes cancer cell lines ${ }^{31,34}$ or untransformed cells with ectopic oncogene expression to the effects of specific environmental conditions, like a drug.

Recently, in synthetic lethality screens using shRNAs in human cancer cells expressing mutant K-RAS, Gilliland and colleagues and Elledge and colleagues identified 2 different kinases-STK33 (serine/threonine kinase 33) and PLK1 (polo-like kinase 1)-respectively, as potential novel drug targets for RAS-driven cancers. ${ }^{18,25}$ In a panel of 8 human cancer cell lines ( $4 K-R A S$ wild-type and 4 $K-R A S$ mutant), using a well-by-well approach in which the biological effects of each hairpin are scored individually, Gilliland and colleagues screened 5000 shRNAs targeting 1000 genes. Annotation of the hits that scored 
specifically in the $4 K$-RAS mutant lines yielded a small list of genes, which included STK33 at the top of the list. This gene was validated in separate in vitro assays, and in vivo in tumor xenograft models. This large-scale, wellby-well screening approach requires an expensive highthroughput platform that, while powerful, would likely be difficult for most other academic labs to recapitulate. Scaling such technology for genome-wide screens would also be challenging.

Elledge and colleagues use a pooled screening approach with an isogenic cell line pair. In their screen, a library of 75,000 shRNAs targeting 30,000 mRNA transcripts from 12,000 genes was introduced into a $K-R A S$ mutant cancer cell line as well as an isogenic wild-type $K-R A S$ control. ${ }^{18}$ As detected by barcode arrays, shRNAs targeting 400 genes were selectively depleted in $K-R A S$ mutant cells after multiple population doublings. These genes were deemed candidate $R A S$ synthetic lethal genes. A subset of these were validated in a repeat screen in another $K-R A S$ mutant isogenic cell line pair, resulting in a list of 77 validated $R A S$ synthetic lethal genes. Due to the larger scale of the screening approach, no single gene was classified as a top hit; however, computational analysis revealed increased dependence of $K-R A S$ mutant cells on multiple genes involved in mitotic machinery (including the kinase gene PLK1) and the proteasome. Consistent with these results, $K-R A S$ mutant cells were preferentially killed by drugs like paclitaxel (which target mitotic spindle function), a preclinical PLK1 inhibitor, and bortezomib (a proteasome inhibitor). These data also bolstered the notion that cancer cells must adapt to avoid stressinduced death associated with their actively proliferating state. Standard chemical approaches should be able to identify inhibitors of these kinases. Ultimately, validation of the synthetic lethal screening strategies outlined will be achieved if patients with $K$-RAS mutant tumors benefit from treatment with STK33 or PLK1 inhibitors.

\section{Future Directions}

Within a short time of its discovery, RNAi has emerged as the preferred means for studying gene function. Marriage of RNAi to high-throughput screening technologies has revolutionized biological and biomedical investigations. Applications of genome-wide RNAi screens to GBM will undoubtedly unveil genes critical for tumor initiation and vulnerabilities inherent within the cancer cells. The hybridization of this information with the genomic landscape as characterized by the sequencing efforts will yield novel insights into glioblastoma pathogenesis and shape strategies for therapeutic development.

\section{Disclosure}

The authors report no conflict of interest concerning the materials or methods used in this study or the findings specified in this paper.

Author contributions to the study and manuscript preparation include the following. Conception and design: CC Chen, KT Kahle, D Kozono. Drafting the article: KT Kahle, D Kozono, G Hsieh, PO Zinn. Critically revising the article: CC Chen, KT Kahle, D Kozono,
K Ng, G Hsieh, PO Zinn, M Nitta. Reviewed final version of manuscript and approved it for submission: CC Chen.

\section{References}

1. Aza-Blanc P, Cooper CL, Wagner K, Batalov S, Deveraux QL, Cooke MP: Identification of modulators of TRAIL-induced apoptosis via RNAi-based phenotypic screening. Mol Cell 12:627-637, 2003

2. Bender A, Pringle JR: Use of a screen for synthetic lethal and multicopy suppressee mutants to identify two new genes involved in morphogenesis in Saccharomyces cerevisiae. Mol Cell Biol 11:1295-1305, 1991

3. Berns K, Hijmans EM, Mullenders J, Brummelkamp TR, Velds A, Heimerikx M, et al: A large-scale RNAi screen in human cells identifies new components of the p53 pathway. Nature 428:431-437, 2004

4. Berns K, Horlings HM, Hennessy BT, Madiredjo M, Hijmans EM, Beelen K, et al: A functional genetic approach identifies the PI3K pathway as a major determinant of trastuzumab resistance in breast cancer. Cancer Cell 12:395-402, 2007

5. Brummelkamp TR, Fabius AW, Mullenders J, Madiredjo M, Velds A, Kerkhoven RM, et al: An shRNA barcode screen provides insight into cancer cell vulnerability to MDM2 inhibitors. Nat Chem Biol 2:202-206, 2006

6. Bui T, Thompson CB: Cancer's sweet tooth. Cancer Cell 9: 419-420, 2006

7. Cancer Genome Atlas Research Network: Comprehensive genomic characterization defines human glioblastoma genes and core pathways. Nature 455:1061-1068, 2008

8. Gazdar AF: Personalized medicine and inhibition of EGFR signaling in lung cancer. N Engl J Med 361:1018-1020, 2009

9. Hartwell LH, Szankasi P, Roberts CJ, Murray AW, Friend SH: Integrating genetic approaches into the discovery of anticancer drugs. Science 278:1064-1068, 1997

10. Kaelin WG Jr: The concept of synthetic lethality in the context of anticancer therapy. Nat Rev Cancer 5:689-698, 2005

11. Kittler R, Buchholz F: Functional genomic analysis of cell division by endoribonuclease-prepared siRNAs. Cell Cycle 4:564-567, 2005

12. Kolfschoten IG, van Leeuwen B, Berns K, Mullenders J, Beijersbergen RL, Bernards R, et al: A genetic screen identifies PITX1 as a suppressor of RAS activity and tumorigenicity. Cell 121:849-858, 2005

13. Liang Y, Diehn M, Watson N, Bollen AW, Aldape KD, Nicholas MK, et al: Gene expression profiling reveals molecularly and clinically distinct subtypes of glioblastoma multiforme. Proc Natl Acad Sci U S A 102:5814-5819, 2005

14. Louis DN: Molecular pathology of malignant gliomas. Annu Rev Pathol 1:97-117, 2006

15. Louis DN, Ohgaki H, Wiestler OD, Cavenee WK, Burger PC, Jouvet A, et al: The 2007 WHO classification of tumours of the central nervous system. Acta Neuropathol 114:97-109, 2007

16. Lucchesi JC: Synthetic lethality and semi-lethality among functionally related mutants of Drosophila melanfgaster. Genetics 59:37-44, 1968

17. Luo B, Cheung HW, Subramanian A, Sharifnia T, Okamoto M, Yang X, et al: Highly parallel identification of essential genes in cancer cells. Proc Natl Acad Sci U S A 105:2038020385, 2008

18. Luo J, Emanuele MJ, Li D, Creighton CJ, Schlabach MR, Westbrook TF, et al: A genome-wide RNAi screen identifies multiple synthetic lethal interactions with the Ras oncogene. Cell 137:835-848, 2009

19. Ngo VN, Davis RE, Lamy L, Yu X, Zhao H, Lenz G, et al: A loss-of-function RNA interference screen for molecular targets in cancer. Nature 441:106-110, 2006 


\section{Functional genomics}

20. Paddison PJ, Silva JM, Conklin DS, Schlabach M, Li M, Aruleba S, et al: A resource for large-scale RNA-interferencebased screens in mammals. Nature 428:427-431, 2004

21. Parsons DW, Jones S, Zhang X, Lin JC, Leary RJ, Angenendt $\mathrm{P}$, et al: An integrated genomic analysis of human glioblastoma multiforme. Science 321:1807-1812, 2008

22. Phillips HS, Kharbanda S, Chen R, Forrest WF, Soriano RH, Wu TD, et al: Molecular subclasses of high-grade glioma predict prognosis, delineate a pattern of disease progression, and resemble stages in neurogenesis. Cancer Cell 9:157-173, 2006

23. Root DE, Hacohen N, Hahn WC, Lander ES, Sabatini DM: Genome-scale loss-of-function screening with a lentiviral RNAi library. Nat Methods 3:715-719, 2006

24. Schlabach MR, Luo J, Solimini NL, Hu G, Xu Q, Li MZ, et al: Cancer proliferation gene discovery through functional genomics. Science 319:620-624, 2008

25. Scholl C, Fröhling S, Dunn IF, Schinzel AC, Barbie DA, Kim SY, et al: Synthetic lethal interaction between oncogenic KRAS dependency and STK33 suppression in human cancer cells. Cell 137:821-834, 2009

26. Silva JM, Li MZ, Chang K, Ge W, Golding MC, Rickles RJ, et al: Second-generation shRNA libraries covering the mouse and human genomes. Nat Genet 37:1281-1288, 2005

27. Silva JM, Marran K, Parker JS, Silva J, Golding M, Schlabach MR, et al: Profiling essential genes in human mammary cells by multiplex RNAi screening. Science 319:617-620, 2008

28. Simons A, Dafni N, Dotan I, Oron Y, Canaani D: Establishment of a chemical synthetic lethality screen in cultured human cells. Genome Res 11:266-273, 2001

29. Solimini NL, Luo J, Elledge SJ: Non-oncogene addiction and the stress phenotype of cancer cells. Cell 130:986-988, 2007
30. Stockwell BR, Haggarty SJ, Schreiber SL: High-throughput screening of small molecules in miniaturized mammalian cell-based assays involving post-translational modifications. Chem Biol 6:71-83, 1999

31. Turner NC, Lord CJ, Iorns E, Brough R, Swift S, Elliott R, et al: A synthetic lethal siRNA screen identifying genes mediating sensitivity to a PARP inhibitor. EMBO J 27:1368-1377, 2008

32. Weinstein IB: Cancer. Addiction to oncogenes-the Achilles heal of cancer. Science 297:63-64, 2002

33. Westbrook TF, Stegmeier F, Elledge SJ: Dissecting cancer pathways and vulnerabilities with RNAi. Cold Spring Harb Symp Quant Biol 70:435-444, 2005

34. Whitehurst AW, Bodemann BO, Cardenas J, Ferguson D, Girard L, Peyton M, et al: Synthetic lethal screen identification of chemosensitizer loci in cancer cells. Nature 446:815-819, 2007

35. Whitesell L, Lindquist SL: HSP90 and the chaperoning of cancer. Nat Rev Cancer 5:761-772, 2005

36. Yan H, Parsons DW, Jin G, McLendon R, Rasheed BA, Yuan W, et al: IDH1 and IDH2 mutations in gliomas. N Engl J Med 360:765-773, 2009

Manuscript submitted September 15, 2009.

Accepted October 22, 2009.

Address correspondence to: Clark C. Chen, M.D., Ph.D., DanaFarber Cancer Institute, Department of Radiation Oncology, Harvard Medical School, 44 Binney Street, Boston, Massachusetts 02115. email: clark_chen@dfci.harvard.edu. 